(C) 2018 IEEE. Personal use of this material is permitted. Permission from IEEE must be obtained for all other uses, in any current or future media, including reprinting/republishing this material for advertising or promotional purposes, creating new collective works, for resale or redistribution to servers or lists, or reuse of any copyrighted component of this work in other works. 


\title{
Frequency Domain Characterization and Performance Bounds of ALMS Loop for RF Self-Interference Cancellation
}

\author{
Anh Tuyen Le, Le Chung Tran, Senior Member, IEEE, Xiaojing Huang, Senior Member, IEEE, \\ Y. Jay Guo, Fellow, IEEE, and J. (Yiannis) C. Vardaxoglou, Fellow, IEEE
}

\begin{abstract}
Analog Least Mean Square (ALMS) loop is a promising method to cancel self-interference (SI) in in-band fullduplex (IBFD) systems. In this paper, the steady state analyses of the residual SI powers in both analog and digital domains are firstly derived. Eigenvalue decomposition is then utilized to investigate the frequency domain characteristics of the ALMS loop. Our frequency domain analyses prove that the ALMS loop has an effect of amplifying the frequency components of the residual SI at the edges of the signal spectrum in the analog domain. However, the matched filter in the receiver chain will reduce this effect, resulting in a significant improvement of the interference suppression ratio (ISR). It means that the SI will be significantly suppressed in the digital domain before information data detection. This paper also derives the lower bounds of ISRs given by the ALMS loop in both analog and digital domains. These lower bounds are joint effects of the loop gain, tap delay, number of taps, and transmitted signal properties. The discovered relationship among these parameters allows the flexibility in choosing appropriate parameters when designing the IBFD systems under given constraints.
\end{abstract}

Index Terms-IBFD, self-interference cancellation, ALMS loop, frequency-domain analysis, matched filter, and eigenvalue decomposition.

\section{INTRODUCTION}

$\mathbf{S}$ Pectral efficiency is always a critical issue in wireless communications as the number of mobile devices has been booming recently. In-band full-duplex (IBFD) transmission is a promising solution for this problem because it allows simultaneous transmission and reception in the same frequency band [1]. Moreover, IBFD transmission provides other benefits, such as avoiding collision due to hidden terminal problems in carrier sense multiple access networks and reducing the endto-end delay in multi-hop networks [2]. However, a critical challenge encountered in implementing IBFD transceivers is that the strong self-interference (SI) imposed by the transmitter prevents its co-located receiver from receiving the signal of interest emitted from the far-end. Hence, SI cancellation (SIC) is a fundamental issue in IBFD communications.

A. T. Le, X. Huang and Y. Jay Guo are with Faculty of Engineering and IT, University of Technology Sydney, Ultimo, NSW, 2007, Australia (emails: anhtuyen.le@student.uts.edu.au, xiaojing.huang@uts.edu.au, and jay.guo@uts.edu.au).

L. C. Tran is with Faculty of Engineering and Information Sciences, University of Wollongong, Wollongong, NSW, 2522 Australia (email: lctran@uow.edu.au).

J. C. Vardaxoglou is with Wolfson School of Mechanical, Electrical and Manufacturing Engineering, Loughborough University, Loughborough, Leicestershire, LE11 3TU, UK (email: j.c.vardaxoglou@lboro.ac.uk).
Numerous approaches have been proposed in the literature to tackle the problem of SI. These approaches can be classified as passive suppression, analog cancellation, and digital cancellation [3]. Passive suppression methods intend to attenuate the level of SI in the propagation domain by separating transmit and receive antennas [4]-[6], or using a circulator to share one antenna [7], [8]. Analog cancellation attempts to generate a reference signal which is a replica of the SI to subtract it from the received signal at the input of the receiver. Digital cancellation is implemented after the Analog-to-Digital converter (ADC) where the residual SI is estimated and subtracted from the received digital signal samples [5]. Note that no single method of cancellation can be sufficient to remove the effect of the SI, but a combination of them is always required [2]. However, analog cancellation plays a critical role in the above mentioned three steps of mitigating the SI. The reason is that passive suppression is limited by the device size, and the level of suppression is not sufficient to protect the ADC from being saturated by the strong SI. As a result, the digital cancellation cannot be solely implemented without the analog domain cancellation. Among many different analog domain SIC techniques, the radio frequency (RF) multi-tap finite impulse response (FIR) adaptive filtering approach [9], the multiple RF bandpass filter (BPF) approach [10], and the RF FIR frequency-domain equalization approach [11] are some of the notable ones. The approaches proposed in [10], [11] directly synthesize the frequency domain characteristics of the SI channel, but the RF BPFs and FIR filter are all static though they can be reconfigurable. Due to practical impairments, such as nonlinearity of the transmit power amplifier (PA), as well as the variation of the SI channel, an adaptive mechanism which can adjust the phase and amplitude of the cancellation signal seems more effective.

An obvious problem here is how to synthesize the weighting coefficients of the multi-tap adaptive filter in order to minimize the power of the residual SI after cancellation. A promising method is to utilize a least mean square (LMS) loop in the adaptive filter. Unlike conventional LMS algorithms in the digital domain, it is very challenging to implement an LMS loop in the RF domain due to the lack of RF integrators. Therefore, many existing SIC filters implement the LMS algorithm at the baseband stage. Besides the baseband integrator, additional down-conversion and ADC circuits have to be added to digitize the residual SI for the LMS filter in baseband 
[3], [9], [12], [13]. Unfortunately, these additional blocks not only consume more power, but also produce further noise and interference to the receiver. Other SIC methods synthesize the weighting coefficients from the digitalized residual SI after the $\mathrm{ADC}$ in the receiver chain and generate the RF cancellation signal by an additional transmit chain [14]-[16]. However, in a conventional receiver, an automatic gain control (AGC) amplifier is always required to avoid the problem of fading and ensure the wide dynamic range of the receiver. Since the level of residual SI is stabilized by the AGC amplifier, the weight coefficients synthesized in the digital domain are inaccurate. Furthermore, the involvement of the transmitted baseband signal in the control algorithm also makes the cancellation circuit become more complicated in practice.

A novel analog LMS (ALMS) loop purely implemented at the RF stage is proposed in [17]. By employing a simple resistor-capacitor low-pass filter (LPF) to replace the ideal integrator, the weighting coefficients can be synthesized without any involvement of the complicated digital signal processing. The performance and convergence of the ALMS loop are comprehensively investigated by examining the weighting error function in both micro and macro scales. The spectra of residual SI obtained from experiment results show that the ALMS loop enhances the SI at the two edges of the signal spectrum. However, this phenomenon has not yet been analyzed and its impact on the SIC performance is not fully understood. As further studied in [18], [19], the properties of transmitted signals have significant impacts on the performance of the ALMS loop, but the roles of the tap delay and the number of taps in ALMS loop in relation to the SIC performance have not been considered. As we all know, as long as the level of passive suppression and analog cancellation is sufficient to allow the received signal to be digitized within the ADC's dynamic range, the SIC performance in the RF stage does not show the real impact on the performance of information detection since further optimal receiver algorithms including matched filtering and equalization will be performed in the digital domain. Therefore, it would make more sense to consider the performance of the ALMS loop in the digital domain after the matched filter. However, the analyses on ALMS loop performance in [17]-[19] are all conducted at the RF stage.

To overcome the aforementioned shortcoming, in this paper, we analyze the performance of the ALMS loop proposed in [17] by evaluating the interference-suppression-ratios (ISRs) in both analog and digital domains in the receiver chain. In particular, the ISRs before and after the matched filter are firstly derived by a steady state analysis, and eigenvalue decomposition is then performed to derive the frequency domain presentation of the ALMS loop. We prove that although the ALMS loop has an effect of amplifying the frequency components of the residual SI at the edges of the signal spectrum, this effect is significantly reduced by the matched filter, leading to a much lower ISR at the output of the matched filter. Hence, unlike [17], the real effect of the ALMS loop on the SI suppression should be considered after the matched filter in the digital domain instead of before it in the analog domain. Furthermore, the lower bounds of ISRs in both analog and digital domains are derived to characterize the performance of the ALMS loop with regards to the transmitted signal property, the loop gain, the tap spacing, and the number of taps. From the relationship among these parameters, the full potential of SIC given by the ALMS loop can be determined.

Contributions of this paper are twofold. First, this paper characterizes the phenomenon of frequency component enhancement produced by the ALMS loop to the residual SI, and proves mathematically that the matched filter reduces this enhancement, leading to a significant improvement of ISR in the digital domain. Second, the lower bound of ISR given by the ALMS loop in the digital domain derived in this paper allows the designer to determine the expected level of suppression from the parameters of the transceiver and the cancellation circuit. More importantly, this expected level can be achieved by adjusting the remaining parameters when others are under constraints.

The rest of this paper is organized as follows. Section II describes the system architecture and the signal models and performs the steady state analysis to find the expressions of ISRs in both analog and digital domains. In Section III, the ISRs are analyzed in the frequency domain and their lower bounds are derived respectively. In Section IV, simulations are conducted to verify the theoretical findings. Finally, conclusions are drawn in Section $\mathrm{V}$.

\section{STEADY STATE ANALYSIS OF ALMS LOOP}

\section{A. IBFD Transceiver with ALMS LOOP}

The architecture of an IBFD transceiver employing an ALMS loop in the analog domain proposed in [17] is shown in Fig. 1. The ALMS loop works as follows. A copy of the transmitted signal is passed through the ALMS loop, which includes $L$ taps. In each tap, the transmitted signal is delayed and multiplied by the amplified and looped-back residual SI with an I/Q demodulator. This product is then filtered with the LPFs to obtain the weighting coefficient $w_{l}(t)$. These weighting coefficients modulate again the same delayed transmitted signal. The outputs of the $L$-taps are added together to produce the cancellation signal $y(t)$, which is then subtracted from the received signal $r(t)$ at the input of the receiver.

Signal models are described as follows. Assuming a single carrier system, the transmitted signal $x(t)$ at the output of the power amplifier (PA) is modeled as $x(t)=\operatorname{Re}\left\{X(t) e^{j 2 \pi f_{c} t}\right\}$ where $f_{c}$ is the carrier frequency, and $X(t)$ is the baseband equivalent which can be mathematically modeled as

$$
X(t)=\sum_{i=-\infty}^{\infty} a_{i} V_{X} p\left(t-i T_{s}\right)
$$

where $a_{i}$ is the $i$-th complex data symbol, $T_{S}$ is the symbol interval, $V_{X}$ is the root mean square (RMS) value of the transmitted signal, and $p(t)$ is the pulse shaping function with unit power $\frac{1}{T s} \int_{0}^{T_{s}}|p(t)|^{2} d t=1$. The transmitted data symbols $a_{i}$ are assumed to be independent of each other, i.e., $E\left\{a_{i}^{*} a_{i^{\prime}}\right\}=\left\{\begin{array}{l}1, \text { for } i=i^{\prime} \\ 0, \text { for } i \neq i^{\prime}\end{array}\right.$ where $E\{$.$\} stands for en-$ semble expectation. The average power of $X(t)$ is defined as 


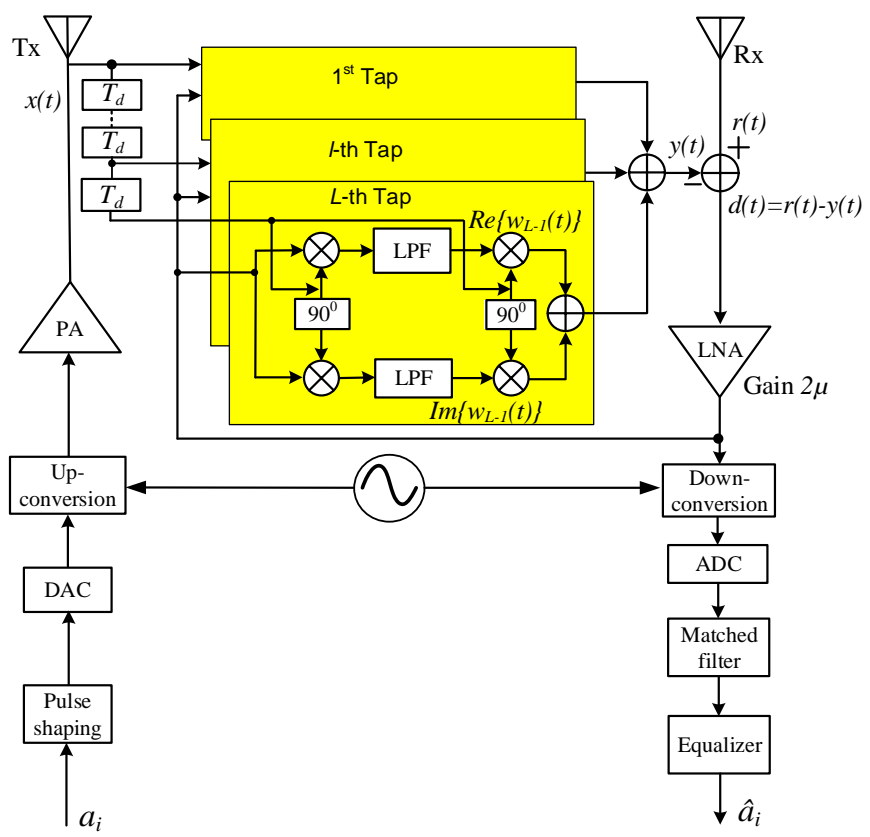

Fig. 1. The ALMS loop structure.

$\frac{1}{T_{s}} \int_{0}^{T_{s}} E\left\{|X(t)|^{2}\right\} d t=V_{X}^{2}$ over $1 \Omega$ load. Due to the IBFD operation, at the input of the receiver, there are presences of the SI $z(t)$, the desired signal $s(t)$, and the additive Gaussian noise $n(t)$, i.e., $r(t)=z(t)+s(t)+n(t)$. The baseband equivalents of these signals are denoted as $R(t), Z(t), S(t)$ and $N(t)$ respectively. The cancellation signal $y(t)$ is combined from the $L$ taps as

$$
y(t)=\operatorname{Re}\left\{\sum_{l=0}^{L-1} w_{l}^{*}(t) X\left(t-l T_{d}\right) e^{j 2 \pi f_{c}\left(t-l T_{d}\right)}\right\}
$$

where $w_{l}(t)$ is the complex weighting coefficient at the $l$-th tap obtained by filtering the outputs of the I/Q demodulator, $T_{d}$ is the delay between adjacent taps. As proved in [17], using a simple resistor-capacitor LPF with the decay constant $\alpha(\alpha=$ $1 / R C)$, the weighting coefficients $w_{l}(t)$ can be written as

$$
w_{l}(t)=\frac{2 \mu \alpha}{K_{1} K_{2}} \int_{0}^{t} e^{-\alpha(t-\tau)}[r(\tau)-y(\tau)] \cdot X\left(\tau-l T_{d}\right) e^{j 2 \pi f_{c}\left(\tau-l T_{d}\right)} d \tau
$$

where $K_{1}$ and $K_{2}$ are the dimensional constants of multipliers in the I/Q demodulator and I/Q modulator respectively, and $2 \mu$ is the gain of the low noise amplifier (LNA). Assume that the SI channel is modeled as an $L$-stage multi-tap filter where each tap has a coefficient $h_{l}^{*}$ and delay $T_{d}$. Hence, the baseband equivalent of the SI $z(t)$ can be expressed as $Z(t)=\sum_{l=0}^{L-1} h_{l}^{*} X\left(t-l T_{d}\right)$. Obviously, the performance of the ALMS loop is determined by the difference between the cancellation signal $y(t)$ and the SI $z(t)$. This difference is represented by the weighting error function defined as

$$
u_{l}(t)=h_{l}-w_{l}(t) e^{j 2 \pi f_{c} l T_{d}} .
$$

As derived in [17, Eq.(11)], $u_{l}(t)$ can be expressed as

$$
\begin{aligned}
u_{l}(t) & =h_{l}-\frac{\mu \alpha}{K_{1} K_{2}} \int_{0}^{t} e^{-\alpha(t-\tau)}\left[\sum_{l^{\prime}=0}^{L-1} u_{l^{\prime}}(\tau) X^{*}\left(\tau-l^{\prime} T_{d}\right)\right. \\
& \left.+S^{*}(\tau)+N^{*}(\tau)\right] X\left(\tau-l T_{d}\right) d \tau .
\end{aligned}
$$

\section{B. Steady State Analysis}

1) Steady State of Weighting Error Function: Now we apply the steady state analysis to derive the residual SI power and the ISR at the output of the ALMS loop. The system is assumed to be steady after an initial start-up so that all the weighting coefficients are in their converged values. Both ensemble expectation and time averaging denoted as $\bar{E}\{$.$\} are$ used to evaluate the random processes involved in this analysis. The normalized autocorrelation function of the transmitted signal is defined by

$$
\begin{aligned}
\Phi(\tau) & =\frac{1}{K_{1} K_{2}} \bar{E}\left\{X^{*}(t) X(t-\tau)\right\} \\
& =\frac{1}{K_{1} K_{2} T_{s}} \int_{0}^{T_{s}} E\left\{X^{*}(t) X(t-\tau)\right\} d t \\
& =\frac{V_{X}^{2}}{K_{1} K_{2} T_{s}} \int_{-\infty}^{\infty} p^{*}(t) p(t-\tau) d t \\
& =\frac{A^{2}}{T_{S}} \int_{-\infty}^{\infty} p^{*}(t) p(t-\tau) d t
\end{aligned}
$$

where $A^{2}=V_{X}^{2} / K_{1} K_{2}=\Phi(0)$ is the normalized power of the transmitted signal. To simplify (5), we assume that the transmitted signal is independent of the desired signal and the additive Gaussian noise, i.e., $\bar{E}\left\{S^{*}(t) X(t-\tau)\right\}=0$ and $\bar{E}\left\{N^{*}(t) X(t-\tau)\right\}=0$ for all $\tau$. Performing both ensemble expectation and time averaging and applying the above assumptions to (5), we have

$$
\overline{\bar{u}}_{l}(t)=h_{l}-\mu \alpha \int_{0}^{t} e^{-\alpha(t-\tau)} \sum_{l^{\prime}=0}^{L-1} \overline{\bar{u}}_{l^{\prime}}(\tau) \Phi\left(\left(l-l^{\prime}\right) T_{d}\right) d \tau,
$$

or, in matrix form

$$
\overline{\overline{\mathbf{u}}}(t)=\mathbf{h}-\mu \alpha \int_{0}^{t} e^{-\alpha(t-\tau)} \boldsymbol{\Phi} \overline{\overline{\mathbf{u}}}(t) d \tau
$$

where $\overline{\bar{u}}_{l}(t)=\bar{E}\left\{u_{l}(t)\right\}, \overline{\overline{\mathbf{u}}}(t)=\left[\overline{\bar{u}}_{0}(t), \quad \overline{\bar{u}}_{1}(t) \cdots \overline{\bar{u}}_{L-1}(t)\right]^{H}, \mathbf{h}=$ $\left[h_{0}, h_{1}, \cdots, h_{L-1}\right]^{H}$, and

$\boldsymbol{\Phi}=\left[\begin{array}{cccc}\Phi(0) & \Phi\left(-T_{d}\right) & \cdots & \Phi\left(-(L-1) T_{d}\right) \\ \Phi\left(T_{d}\right) & \Phi(0) & \cdots & \Phi\left(-(L-2) T_{d}\right) \\ \vdots & \vdots & \ddots & \vdots \\ \Phi\left((L-1) T_{d}\right) & \Phi\left((L-2) T_{d}\right) & \cdots & \Phi(0)\end{array}\right]$. When $t \rightarrow \infty, \overline{\overline{\mathbf{u}}}(t)$ converge to their steady-state values $\overline{\overline{\mathbf{u}}}$ so that $\overline{\overline{\mathbf{u}}}(t)$ can be taken out of the integral in (8). It is also noted that $\left.\alpha \int_{0}^{t} e^{-\alpha(t-\tau)} d \tau\right|_{t \rightarrow \infty} \rightarrow 1$. Therefore, (8) becomes

$$
\overline{\overline{\mathbf{u}}}=\mathbf{h}-\mu \boldsymbol{\Phi} \overline{\overline{\mathbf{u}}}
$$

and hence

$$
\overline{\overline{\mathbf{u}}}=\left(\mathbf{I}_{L}+\mu \boldsymbol{\Phi}\right)^{-1} \mathbf{h} .
$$


2) Interference Suppression Ratios: ISR is an important metric to evaluate the performance of the cancellation circuit. In this subsection, we derive the closed-form equations of ISRs before and after the matched filter in the analog domain and digital domain respectively.

a) ISR in analog domain: After SI cancellation, the normalized power of residual SI $v(t)=z(t)-y(t)$ is derived as

$$
\begin{aligned}
& P_{v}(t)=\frac{1}{K_{1} K_{2}} \bar{E}\left\{[z(t)-y(t)]^{2}\right\} \\
& =\frac{1}{K_{1} K_{2}} \bar{E}\left\{\left[\operatorname{Re}\left\{\left[Z(t)-\sum_{l=0}^{L-1}\left(h_{l}^{*}-u_{l}^{*}(t)\right) X\left(t-l T_{d}\right)\right] e^{j 2 \pi f_{c} t}\right\}\right]^{2}\right\} \\
& =\frac{1}{2 K_{1} K_{2}} \bar{E}\left\{\left|Z(t)-\sum_{l=0}^{L-1}\left(h_{l}^{*}-u_{l}^{*}(t)\right) X\left(t-l T_{d}\right)\right|^{2}\right\} \\
& =\frac{1}{2 K_{1} K_{2}} \bar{E}\left\{\left[\sum_{l=0}^{L-1} u_{l}^{*}(t) X\left(t-l T_{d}\right) \sum_{l^{\prime}=0}^{L-1} u_{l^{\prime}}(t) X^{*}\left(t-l^{\prime} T_{d}\right)\right]\right\} \\
& =\frac{1}{2} \bar{E}\left\{\sum_{l=0}^{L-1} \sum_{l^{\prime}=0 ; l^{\prime} \neq l}^{L-1} u_{l}^{*}(t) \Phi\left(\left(l-l^{\prime}\right) T_{d}\right) u_{l^{\prime}}(t)+\Phi(0) \sum_{l=0}^{L-1}\left|u_{l}(t)\right|^{2}\right\} \\
& =\frac{1}{2} \overline{\overline{\mathbf{u}}}^{H}(t)\left[\boldsymbol{\Phi}-\Phi(0) \mathbf{I}_{L}\right] \overline{\overline{\mathbf{u}}}(t)+\frac{1}{2} \Phi(0) \sum_{l=0}^{L-1} \overline{\bar{u}}_{l}^{2}(t)
\end{aligned}
$$

where $\overline{\bar{u}}_{l}^{2}(t)=\bar{E}\left\{\left|u_{l}(t)\right|^{2}\right\}$ is the time-averaged mean square value of $u_{l}(t)$. From (5), following the steps shown in Appendix B in [17], when $\frac{d \overline{\bar{u}}_{l}^{2}(t)}{d t}=0, \overline{\bar{u}}_{l}^{2}(t)$ satisfies the equation

$$
\left(1+\mu A^{2}\right) \sum_{l=0}^{L-1} \overline{\bar{u}}_{l}^{2}(t)=\operatorname{Re}\left\{\overline{\overline{\mathbf{u}}}^{H} \mathbf{h}\right\}-\mu \overline{\overline{\mathbf{u}}}^{H}\left(\boldsymbol{\Phi}-A^{2} \mathbf{I}_{L}\right) \overline{\overline{\mathbf{u}}} .
$$

Substituting (10) to (12), we have

$$
\sum_{l=0}^{L-1} \overline{\bar{u}}_{l}^{2}(t)=\mathbf{h}^{H}\left(\mathbf{I}_{L}+\mu \boldsymbol{\Phi}\right)^{-2} \mathbf{h}
$$

and the steady state power of the residual interference is obtained from (11) as

$$
P_{v}=\frac{1}{2} \mathbf{h}^{H}\left(\mathbf{I}_{L}+\mu \boldsymbol{\Phi}\right)^{-1} \boldsymbol{\Phi}\left(\mathbf{I}_{L}+\mu \boldsymbol{\Phi}\right)^{-1} \mathbf{h} .
$$

If there was no cancellation, the normalized SI power would be

$$
\begin{aligned}
P_{z} & =\frac{1}{K_{1} K_{2}} \bar{E}\left\{[z(t)]^{2}\right\} \\
& =\frac{1}{K_{1} K_{2}} \bar{E}\left\{\left[\operatorname{Re}\left\{\sum_{l=0}^{L-1} h_{l}^{*} X\left(t-l T_{d}\right) e^{j 2 \pi f_{c} t}\right\}\right]^{2}\right\} \\
& =\frac{1}{2 K_{1} K_{2}} \bar{E}\left\{\sum_{l=0}^{L-1} h_{l}^{*} X\left(t-l T_{d}\right) \sum_{l^{\prime}=0}^{L-1} h_{l^{\prime}} X^{*}\left(t-l^{\prime} T_{d}\right)\right\} \\
& =\frac{1}{2 K_{1} K_{2}} \sum_{l=0}^{L-1} \sum_{l^{\prime}=0}^{L-1} h_{l}^{*} \bar{E}\left\{X\left(t-l T_{d}\right) X^{*}\left(t-l^{\prime} T_{d}\right)\right\} h_{l^{\prime}} \\
& =\frac{1}{2} \sum_{l=0}^{L-1} \sum_{l^{\prime}=0}^{L-1} h_{l}^{*} \Phi\left(\left(l-l^{\prime}\right) T_{d}\right) h_{l^{\prime}}=\frac{1}{2} \mathbf{h}^{H} \mathbf{\Phi} \mathbf{h} .
\end{aligned}
$$

Therefore, ISR before the matched filter in the analog domain, denoted as $I S R_{a}$, is determined by

$$
I S R_{a}=\frac{P_{v}}{P_{z}}=\frac{\mathbf{h}^{H}\left(\mathbf{I}_{L}+\mu \boldsymbol{\Phi}\right)^{-1} \boldsymbol{\Phi}\left(\mathbf{I}_{L}+\mu \boldsymbol{\Phi}\right)^{-1} \mathbf{h}}{\mathbf{h}^{H} \boldsymbol{\Phi} \mathbf{h}} .
$$

b) ISR in digital domain: After down-converted to baseband, the residual SI, denoted as $V(t)$, is expressed as

$$
\begin{aligned}
V(t) & =Z(t)-Y(t) \\
& =\sum_{l=0}^{L-1} h_{l}^{*} X\left(t-l T_{d}\right)-\sum_{l=0}^{L-1} w_{l}^{*}(t) X\left(t-l T_{d}\right) e^{-j 2 \pi f_{c} l T_{d}} \\
& =\sum_{l=0}^{L-1} u_{l}^{*}(t) X\left(t-l T_{d}\right)
\end{aligned}
$$

After the matched filter with the impulse response $p^{*}(-t)$, we get the filtered version of $V(t)$ as

$$
\tilde{V}(t)=V(t) * p^{*}(-t)=\sum_{l=0}^{L-1} u_{l}^{*}(t) \tilde{X}\left(t-l T_{d}\right)
$$

where $*$ stands for a linear convolution operation and

$$
\tilde{X}(t)=X(t) * p^{*}(-t)
$$

is the filtered version of the transmitted baseband signal. Similarly, the steady normalized power of the filtered residual SI is calculated as

$$
\begin{aligned}
P_{\tilde{V}} & =\frac{1}{K_{1} K_{2}} \bar{E}\left\{|\tilde{V}(t)|^{2}\right\} \\
& =\frac{1}{K_{1} K_{2}} \bar{E}\left\{\sum_{l=0}^{L-1} u_{l}^{*}(t) \tilde{X}\left(t-l T_{d}\right) \sum_{l^{\prime}=0}^{L-1} u_{l^{\prime}}(t) \tilde{X}^{*}\left(t-l^{\prime} T_{d}\right)\right\} \\
& =\sum_{l=0}^{L-1} \sum_{l^{\prime}=0, l \neq l^{\prime}}^{L-1} \overline{\bar{u}}_{l}^{*}(t) \Theta\left(\left(l-l^{\prime}\right) T_{d}\right) \overline{\bar{u}}_{l^{\prime}}(t)+\Theta(0) \sum_{l=0}^{L-1} \overline{\bar{u}}_{l}^{2}(t) \\
& =\overline{\overline{\mathbf{u}}}^{H}(t)\left(\boldsymbol{\Theta}-\Theta(0) \mathbf{I}_{L}\right) \overline{\overline{\mathbf{u}}}(t)+\Theta(0) \sum_{l=0}^{L-1} \overline{\bar{u}}_{l}^{2}(t) \\
& =\mathbf{h}^{H}\left(\mathbf{I}_{L}+\mu \boldsymbol{\Phi}\right)^{-1} \Theta\left(\mathbf{I}_{L}+\mu \boldsymbol{\Phi}\right)^{-1} \mathbf{h}
\end{aligned}
$$

where $\Theta(\tau)=\frac{1}{K_{1} K_{2}} \bar{E}\left\{\tilde{X}(t) \tilde{X}^{*}(t-\tau)\right\}$ and $\boldsymbol{\Theta}=$

$$
\left[\begin{array}{cccc}
\Theta(0) & \Theta\left(-T_{d}\right) & \cdots & \Theta\left(-(L-1) T_{d}\right) \\
\Theta\left(T_{d}\right) & \Theta(0) & \cdots & \Theta\left(-(L-2) T_{d}\right) \\
\vdots & \vdots & \ddots & \vdots \\
\Theta\left((L-1) T_{d}\right) & \Theta\left((L-2) T_{d}\right) & \cdots & \Theta(0)
\end{array}\right] \quad \text { are }
$$

the normalized autocorrelation function of $\tilde{X}(t)$ and the corresponding autocorrelation matrix respectively.

Meanwhile, if there was no cancellation, the steady normalized SI power after the matched filter would be

$$
\begin{aligned}
P_{\tilde{Z}} & =\frac{1}{K_{1} K_{2}} \bar{E}\left\{\left|Z(t) * p^{*}(-t)\right|^{2}\right\} \\
& =\frac{1}{K_{1} K_{2}} \bar{E}\left\{\left|\sum_{l=0}^{L-1} h_{l}^{*} \tilde{X}\left(t-l T_{d}\right)\right|^{2}\right\} \\
& =\sum_{l=0}^{L-1} \sum_{l^{\prime}=0}^{L-1} h_{l}^{*} \Theta\left(\left(l-l^{\prime}\right) T_{d}\right) h_{l^{\prime}} \\
& =\mathbf{h}^{H} \mathbf{\Theta h} .
\end{aligned}
$$


Therefore, the $I S R$ after the matched filter in the digital domain, denoted as $I S R_{d}$, is

$$
I S R_{d}=\frac{P_{\tilde{V}}}{P_{\tilde{Z}}}=\frac{\mathbf{h}^{H}\left(\mathbf{I}_{L}+\mu \boldsymbol{\Phi}\right)^{-1} \Theta\left(\mathbf{I}_{L}+\mu \boldsymbol{\Phi}\right)^{-1} \mathbf{h}}{\mathbf{h}^{H} \Theta \mathbf{h}} .
$$

\section{FREQUENCY-DOMAIN ANALYSIS OF RESIDUAL SI}

\section{A. Eigen-Decomposition of Autocorrelation Matrices}

The $L \times L$ matrix $\boldsymbol{\Phi}$ can be decomposed as $\boldsymbol{\Phi}=\mathbf{Q} \boldsymbol{\Lambda} \mathbf{Q}^{-1}$ where $\mathbf{Q}$ is the orthonormal modal matrix whose columns are the $L$ eigenvectors of $\boldsymbol{\Phi}$ and $\boldsymbol{\Lambda}=\left(\begin{array}{cccc}\lambda_{0} & 0 & \cdots & 0 \\ 0 & \lambda_{1} & \cdots & 0 \\ \vdots & \vdots & \ddots & \vdots \\ 0 & 0 & \cdots & \lambda_{L-1}\end{array}\right)$ is the spectral matrix whose main diagonal elements are the $L$ eigenvalues of $\boldsymbol{\Phi}$. When $L T_{d}$ is sufficiently large, the autocorrelation matrix $\boldsymbol{\Phi}$ can be approximated as a circulant matrix $\tilde{\boldsymbol{\Phi}}$ composed of a periodic autocorrelation function $\tilde{\Phi}(\tau)=\sum_{l=-\infty}^{\infty} \Phi\left(\tau+l L T_{d}\right)$. As proved in [20], the circulant matrix $\tilde{\boldsymbol{\Phi}}$ can be decomposed as $\tilde{\boldsymbol{\Phi}}=\mathbf{F S}_{\mathbf{X}} \mathbf{F}^{-1}$ where $\mathbf{F}$ is the discrete Fourier transform (DFT) matrix of order $L$, $\mathbf{F}=\left(\begin{array}{cccc}1 & 1 & \cdots & 1 \\ 1 & e^{-j \omega_{1}} & \cdots & e^{-j(L-1) \omega_{1}} \\ \vdots & \vdots & \ddots & \vdots \\ 1 & e^{-j \omega_{L-1}} & \cdots & e^{-j(L-1) \omega_{L-1}}\end{array}\right)$ with $\omega_{k}=\frac{2 \pi k}{L}, k=$ $0,1, \cdots, L-1, \mathbf{S}_{X}=\operatorname{diag}\left\{S_{X}\left(e^{j \omega_{0}}\right), S_{X}\left(e^{j \omega_{1}}\right), \cdots, S_{X}\left(e^{j \omega_{L-1}}\right)\right\}$, and $S_{X}\left(e^{j \omega_{k}}\right)$ are obtained by taking the DFT of $\tilde{\Phi}\left(l T_{d}\right)$, i.e.,

$$
S_{X}\left(e^{j \omega_{k}}\right)=\sum_{l=0}^{L-1} \tilde{\Phi}\left(l T_{d}\right) e^{-j \omega_{k} l}
$$

for $k=0,1, \cdots, L-1$, which are the $\mathrm{L}$ samples of the normalized power spectrum $S_{X}\left(e^{j \omega}\right)$ of the transmitted signal sequence $X\left(n T_{d}\right)$ uniformly spaced about the unit circle. It means that when $L$ is sufficiently large, the eigenvalues $\lambda_{k}$ can be approximated as the power spectrum samples $S_{X}\left(e^{j \omega_{k}}\right)$. To confirm this approximation, the eigenvalues $\lambda_{k}$ are compared with the power spectrum $S_{X}\left(e^{j \omega_{k}}\right)$ as below.

Suppose that the transmitter employs a root raised cosine pulse shaping filter. The autocorrelation function $\Phi(t)$ is a raised cosine pulse, which has the frequency response

$$
P(f)= \begin{cases}T_{s} & \text { for } 0 \leq|f|<\frac{1-\beta}{2 T_{s}} \\ \frac{T_{s}}{2}\left[1+\cos \left(\frac{\pi T_{s}}{\beta}\left(f-\frac{1-\beta}{2 T_{s}}\right)\right)\right] & \text { for } \frac{1-\beta}{2 T_{s}} \leq|f| \leq \frac{1+\beta}{2 T_{s}} \\ 0 & \text { for }|f|>\frac{1+\beta}{2 T_{s}}\end{cases}
$$

where $\beta$ is the roll-off factor. Hence, the normalized power spectrum of $X(t)$ is $A^{2} P(f)$. With the sampling period $T_{d}$, the relationship between $S_{X}\left(e^{j \omega}\right)$ and $P(f)$ can be expressed as

$$
S_{X}\left(e^{j \omega}\right)=\frac{1}{T_{d}} \sum_{n=-\infty}^{\infty} A^{2} P\left(\frac{\omega}{2 \pi T_{d}}-\frac{n}{T_{d}}\right) .
$$

If $T_{d} \leq T_{S} /(1+\beta)$, there will be no spectral overlapping and hence

$$
S_{X}\left(e^{j \omega}\right)=\frac{A^{2}}{T_{d}} P\left(\frac{\omega}{2 \pi T_{d}}\right), \text { for }-\pi<\omega<\pi .
$$
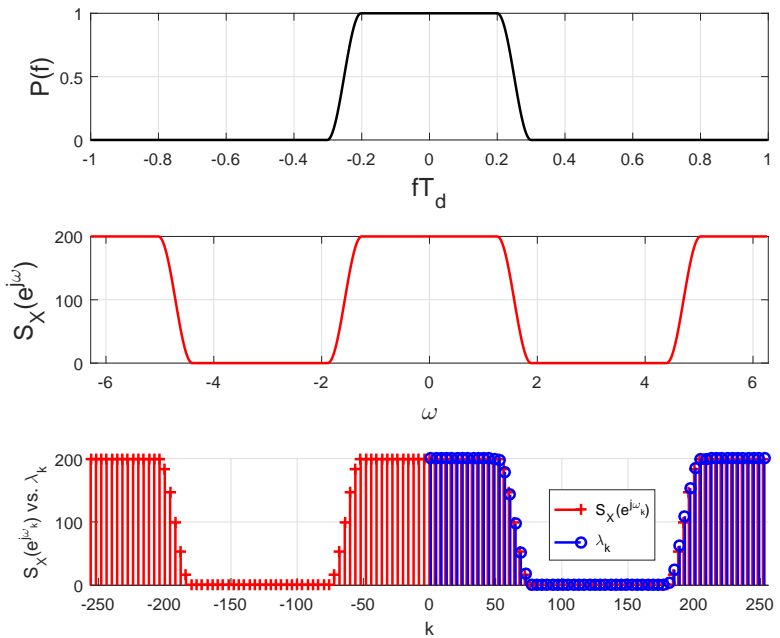

Fig. 2. (a) Raised cosine spectrum; (b) $S_{X}\left(e^{j \omega}\right)$; (c) $S_{X}\left(e^{j \omega_{k}}\right)$ versus eigenvalues $\lambda_{k}$, with $L=256, A^{2}=100, \beta=0.2, T_{d}=T_{s} / 2, T_{s}=1$.

Fig. 2 shows the raised cosine spectrum $P(f), \quad S_{X}\left(e^{j \omega}\right), \quad S_{X}\left(e^{j \omega_{k}}\right)$, and properly ordered $\lambda_{k}$ for $L=256, A^{2}=100, \beta=0.2$, and $T_{d}=T_{s} / 2$ where $T_{s}$ is normalized to 1 . We see that $\lambda_{k}$ are very close to $S_{X}\left(e^{j \omega_{k}}\right)$.

The same approximation can also be applied to the autocorrelation matrix $\boldsymbol{\Theta}$, i.e., it is close to a circulant matrix $\tilde{\boldsymbol{\Theta}}$ when $L$ is sufficiently large. In this case, $\tilde{\boldsymbol{\Theta}}$ can be decomposed as $\tilde{\mathbf{\Theta}}=\mathbf{F S}_{\tilde{X}} \mathbf{F}^{-1}$ where $\mathbf{S}_{\tilde{X}}=$ $\operatorname{diag}\left\{S_{\tilde{X}}\left(e^{j \omega_{0}}\right), S_{\tilde{X}}\left(e^{j \omega_{1}}\right), \cdots, S_{\tilde{X}}\left(e^{j \omega_{L-1}}\right)\right\} ; S_{\tilde{X}}\left(e^{j \omega_{k}}\right)$ for $k=$ $0, \cdots, L-1$ are the $L$ spectrum components obtained by taking DFT of $\tilde{\Theta}\left(l T_{d}\right)$ with $\tilde{\Theta}(\tau)=\sum_{l=-\infty}^{\infty} \Theta\left(\tau+l L T_{d}\right)$, and $S_{\tilde{X}}\left(e^{j \omega}\right)=$ $\frac{A^{2}}{T_{d}} P^{2}\left(\frac{\omega}{2 \pi T_{d}}\right)$ for $-\pi<\omega<\pi$.

\section{B. Frequency Domain Characterization of ALMS Loop}

From the above decomposition, we can simplify (16) and (22) as

$$
\begin{aligned}
I S R_{a}= & \frac{\mathbf{h}^{H} \mathbf{F}\left(\mathbf{I}_{L}+\mu \mathbf{S}_{X}\right)^{-1} \mathbf{F}^{-1} \mathbf{F} \mathbf{S}_{X} \mathbf{F}^{-1} \mathbf{F}\left(\mathbf{I}_{L}+\mu \mathbf{S}_{X}\right)^{-1} \mathbf{F}^{-1} \mathbf{h}}{\mathbf{h}^{H} \mathbf{F} \mathbf{S}_{X} \mathbf{F}^{-1} \mathbf{h}} \\
= & \frac{\mathbf{h}^{H} \mathbf{F} \operatorname{diag}\left\{\frac{S_{X}\left(e^{j \omega_{k}}\right)}{\left[1+\mu S_{X}\left(e^{j \omega_{k}}\right)\right]^{2}}\right\} \mathbf{F}^{-1} \mathbf{h}}{\mathbf{h}^{H} \mathbf{F} \operatorname{diag}\left\{S_{X}\left(e^{j \omega_{k}}\right)\right\} \mathbf{F}^{-1} \mathbf{h}} \\
= & \frac{\sum_{k=0}^{L-1}\left|H\left(e^{i \omega_{k}}\right)\right|^{2} \frac{S_{X}\left(e^{j \omega_{k}}\right)}{\left[1+\mu S_{X}\left(e^{j \omega_{k}}\right)\right]^{2}}}{\sum_{k=0}^{L-1}\left|H\left(e^{i \omega_{k}}\right)\right|^{2} S_{X}\left(e^{j \omega_{k}}\right)}
\end{aligned}
$$




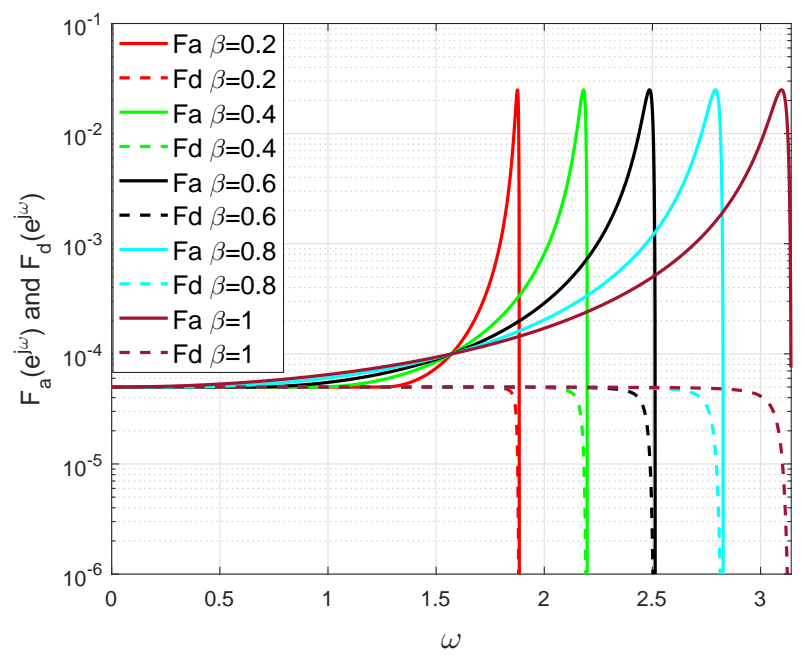

Fig. 3. Frequency dependent attenuation factors with various values of $\beta$, $L=256, A^{2}=100, T_{d}=T_{S} / 2$.

and

$$
\begin{aligned}
I S R_{d} & =\frac{\mathbf{h}^{H} \mathbf{F}\left(\mathbf{I}_{L}+\mu \mathbf{S}_{X}\right)^{-1} \mathbf{F}^{-1} \mathbf{F} \mathbf{S}_{\tilde{X}} \mathbf{F}^{-1} \mathbf{F}\left(\mathbf{I}_{L}+\mu \mathbf{S}_{X}\right)^{-1} \mathbf{F}^{-1} \mathbf{h}}{\mathbf{h}^{H} \mathbf{F} \mathbf{S}_{\tilde{X}} \mathbf{F}^{-1} \mathbf{h}} \\
& =\frac{\mathbf{h}^{H} \mathbf{F} \operatorname{diag}\left\{\frac{S_{\tilde{X}}\left(e^{j \omega_{k}}\right)}{\left[1+\mu S_{X}\left(e^{j \omega_{k}}\right)\right]^{2}}\right\} \mathbf{F}^{-1} \mathbf{h}}{\mathbf{h}^{H} \mathbf{F} \operatorname{diag}\left\{S_{\tilde{X}}\left(e^{j \omega_{k}}\right)\right\} \mathbf{F}^{-1} \mathbf{h}} \\
& =\frac{\sum_{k=0}^{L-1}\left|H\left(e^{j \omega_{k}}\right)\right|^{2} \frac{S_{\tilde{X}}\left(e^{j \omega_{k}}\right)}{\left[1+\mu S_{X}\left(e^{j \omega_{k}}\right)\right]^{2}}}{\sum_{k=0}^{L-1}\left|H\left(e^{j \omega_{k}}\right)\right|^{2} S_{\tilde{X}}\left(e^{j \omega_{k}}\right)}
\end{aligned}
$$

where $H\left(e^{j \omega_{k}}\right)$ is the frequency response of the SI channel. It can be seen from (27) and (28) that, in the frequency domain, the residual SI can be decomposed into two components. The first component is the frequency response of the SI channel $H\left(e^{j \omega_{k}}\right)$. The second component in (27) (i.e., in the analog domain before the matched filter) is a frequency dependent attenuation factor introduced by the ALMS loop as $F_{a}\left(e^{j \omega}\right)=\frac{S_{X}\left(e^{j \omega}\right)}{\left[1+\mu S_{X}\left(e^{j \omega}\right)\right]^{2}}$. Also, in (28), the second component in the digital domain after the matched filter is a frequency dependent attenuation factor determined by both the ALMS loop and the matched filter as $F_{d}\left(e^{j \omega}\right)=\frac{S_{\tilde{X}}\left(e^{j \omega}\right)}{\left[1+\mu S_{X}\left(e^{j \omega}\right)\right]^{2}}$. Therefore, the residual SI before and after the matched filter can be analyzed in the frequency domain by comparing their second components. $F_{a}\left(e^{j \omega}\right)$ and $F_{d}\left(e^{j \omega}\right)$ with various values of $\beta$ are plotted in Fig. 3 respectively.

Fig. 3 reveals that the ALMS loop has an effect of amplifying the frequency components of the residual SI leading to a peak at the edge of the signal spectrum. As a result, the ISR in the analog domain before the matched filter is higher when the roll-off factor is larger. However, this effect is significantly reduced by the matched filter as the peak no longer exists in $F_{d}\left(e^{j \omega}\right)$. Hence, the ISR will be significantly improved in the digital domain. It also means that the effect of the signal spectrum on ISR reduces significantly when it is considered in the digital domain. Therefore, we can conclude that the performance of the ALMS loop evaluated in the digital domain after the matched filter rather than in the analog domain as in [17] makes more sense to the IBFD system.

\section{Performance Lower Bounds}

The ISRs discussed in Section III.A are valid for a given SI channel. To derive the lower bounds of ISRs over random realizations of SI channels, we define the average ISRs in the analog domain and digital domain respectively as

$$
\begin{aligned}
\overline{I S R}_{a} & =\frac{E_{h}\left\{P_{v}\right\}}{E_{h}\left\{P_{z}\right\}}=\frac{\sum_{k=0}^{L-1} E_{h}\left\{\left|H\left(e^{j \omega_{k}}\right)\right|^{2}\right\} \frac{S_{X}\left(e^{j \omega_{k}}\right)}{\left[1+\mu S_{X}\left(e^{j \omega_{k}}\right)\right]^{2}}}{\sum_{k=0}^{L-1} E_{h}\left\{\left|H\left(e^{j \omega_{k}}\right)\right|^{2}\right\} S_{X}\left(e^{j \omega_{k}}\right)} \\
& =\frac{\sum_{k=0}^{L-1} \frac{S_{X}\left(e^{j \omega_{k}}\right)}{\left[1+\mu S_{X}\left(e^{j \omega_{k}}\right)\right]^{2}}}{\sum_{k=0}^{L-1} S_{X}\left(e^{j \omega_{k}}\right)}
\end{aligned}
$$

and

$$
\begin{aligned}
\overline{I S R}_{d}= & \frac{E_{h}\left\{P_{\tilde{V}}\right\}}{E_{h}\left\{P_{\tilde{Z}}\right\}}=\frac{\sum_{k=0}^{L-1} E_{h}\left\{\left|H\left(e^{j \omega_{k}}\right)\right|^{2}\right\} \frac{S_{\tilde{X}}\left(e^{j \omega_{k}}\right)}{\left[1+\mu S_{X}\left(e^{j \omega_{k}}\right)\right]^{2}}}{\sum_{k=0}^{L-1} E_{h}\left\{\left|H\left(e^{j \omega_{k}}\right)\right|^{2}\right\} S_{\tilde{X}}\left(e^{j \omega_{k}}\right)} \\
= & \frac{\sum_{k=0}^{L-1} \frac{S_{\tilde{X}}\left(e^{j \omega_{k}}\right)}{\left[1+\mu S_{X}\left(e^{j \omega_{k}}\right)\right]^{2}}}{\sum_{k=0}^{L-1} S_{\tilde{X}}\left(e^{j \omega_{k}}\right)}
\end{aligned}
$$

where $E_{h}\{$.$\} denotes expectation over the SI channel and$ $E_{h}\left\{\left|H\left(e^{j \omega_{k}}\right)\right|^{2}\right\}$ is a constant for SI channels with independent and zero-mean tap coefficients (see Appendix A). Clearly, $\overline{I S R}_{a}$ and $\overline{I S R}_{d}$ can be purely examined by the spectrum components $S_{X}\left(e^{j \omega_{k}}\right)$ and $S_{\tilde{X}}\left(e^{j \omega_{k}}\right)$. To find the closed-form equation of $\overline{I S R}_{a}$ and $\overline{I S R}_{d}$, letting $L \rightarrow \infty$, the discrete components $S_{X}\left(e^{j \omega_{k}}\right)$ and $\left.S_{\tilde{X}}\left(e^{j \omega_{k}}\right)\right)$ can be replaced by the continuous power spectra $S_{X}\left(e^{j \omega}\right)$ and $S_{\tilde{X}}\left(e^{j \omega}\right)$ respectively. The lower bounds of $\overline{I S R}_{a}$ and $\overline{I S R}_{d}$ are obtained as

$$
\begin{aligned}
I S R L B_{a} & =\left.\overline{I S R}_{a}\right|_{L \rightarrow \infty}=\frac{\frac{1}{2 \pi} \int_{0}^{2 \pi} \frac{S_{X}\left(e^{j \omega}\right)}{\left[1+\mu S_{X}\left(e^{j \omega}\right)\right]^{2}} d \omega}{\frac{1}{2 \pi} \int_{0}^{2 \pi} S_{X}\left(e^{j \omega}\right) d \omega} \\
& =\frac{\frac{1}{2 \pi} \int_{-\pi}^{\pi} \frac{S_{X}\left(e^{j \omega}\right)}{\left[1+\mu S_{X}\left(e^{j \omega}\right)\right]^{2}} d \omega}{\frac{1}{2 \pi} \int_{-\pi}^{\pi} S_{X}\left(e^{j \omega}\right) d \omega} \\
& =\frac{\int_{-1 / 2 T_{d}}^{1 / 2 T_{d}} \frac{A^{2} P(f)}{\left[1+\mu \frac{A^{2}}{T_{d}} P(f)\right]^{2}} d f}{\int_{-1 / 2 T_{d}}^{1 / 2 T_{d}} A^{2} P(f) d f},
\end{aligned}
$$




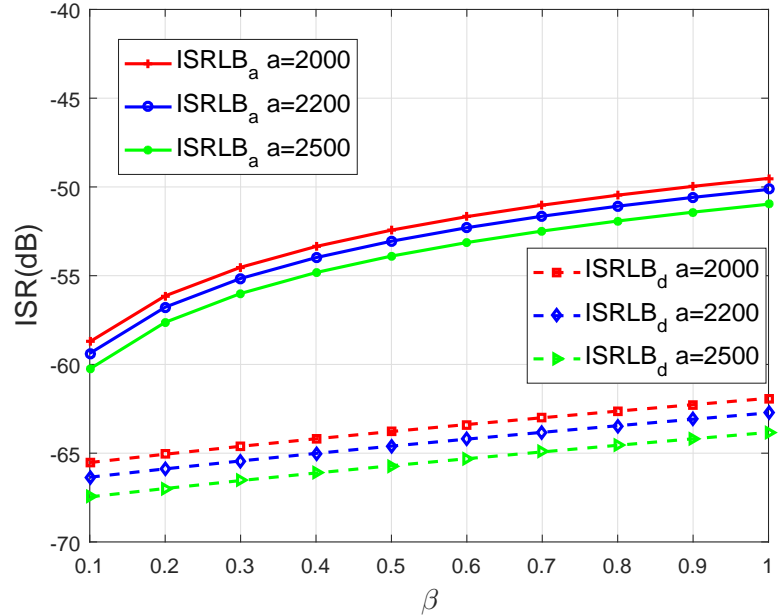

Fig. 4. ISR lower bounds versus $\beta$ with $a=2000,2200$, and 2500 .

and

$$
\begin{aligned}
& I S R L B_{d}=\left.\overline{I S R}_{d}\right|_{L \rightarrow \infty}=\frac{\frac{1}{2 \pi} \int_{0}^{2 \pi} \frac{S_{\tilde{X}}\left(e^{j \omega}\right)}{\left[1+\mu S_{X}\left(e^{j \omega}\right)\right]^{2}} d \omega}{\frac{1}{2 \pi} \int_{0}^{2 \pi} S_{\tilde{X}}\left(e^{j \omega}\right) d \omega} \\
& =\frac{\frac{1}{2 \pi} \int_{-\pi}^{\pi} \frac{S_{\tilde{X}}\left(e^{j \omega}\right)}{\left[1+\mu S_{X}\left(e^{j \omega}\right)\right]^{2}} d \omega}{\frac{1}{2 \pi} \int_{-\pi}^{\pi} S_{\tilde{X}}\left(e^{j \omega}\right) d \omega} \\
& =\frac{\int_{-1 / 2 T_{d}}^{1 / 2 T_{d}} \frac{A^{2} P^{2}(f)}{\left[1+\mu \frac{A^{2}}{T_{d}} P(f)\right]^{2}} d f}{\int_{-1 / 2 T_{d}}^{1 / 2 T_{d}} A^{2} P^{2}(f) d f}
\end{aligned}
$$

respectively. Assuming the raised cosine transmitted signal spectrum, the closed-form $I S R L B_{a}$ and $I S R L B_{d}$ in (31) and (32) are found (see Appendix B) as

$$
\operatorname{ISRLB} B_{a}=\frac{1+\beta(\sqrt{a+1}-1)}{(1+a)^{2}}
$$

and

$$
I S R L B_{d}=\frac{1+\beta\left[\frac{2(a+1)^{2}}{a^{2}}\left(1-\frac{1}{\sqrt{a+1}}-\frac{a \sqrt{a+1}}{2(a+1)^{2}}\right)-1\right]}{(1+a)^{2}(1-\beta / 4)} .
$$

where $a=\mu A^{2} T_{s} / T_{d}$. It is obvious from these lower bounds that in the ideal case $(\beta=0)$ the ultimate level of cancellation is $I S R L B_{u}=1 /\left(1+\frac{T_{s}}{T_{d}} \mu A^{2}\right)^{2}$. Comparison between $I S R L_{a}$ and $I S R L B_{d}$ with various values of $a$ is presented in Fig. 4. From (29), (30), (33), (34), and Fig. 4, some important observations are derived as bellows.

1) The level of cancellation given by the ALMS loop is determined by the loop gain $\mu A^{2}$, the roll-off factor $\beta$ the tap delay $T_{d}$, and the number of taps $L$. It means that the expected level of cancellation can be achieved by either increasing the loop gain $\mu A^{2}$ or reducing the tap delay $T_{d}$. However for the latter case, we need larger number of taps $L$ so that $L T_{d}$ is sufficiently large and $I S R_{a}$ can approach its lower bound.
2) ISRLB $B_{a}$ increases significantly as the roll-off factor increases. As shown in Fig. 4, ISRLB $B_{a}$ for $\beta=1$ is about $10 \mathrm{~dB}$ higher than that for $\beta=0.1$. However, the difference in $I S R L B_{d}$ is only about $3 \mathrm{~dB}$ over the whole range of $\beta$. This indicates that the matched filter significantly reduces the effects of the roll-off factor and the impact of the spectrum of the transmitted signal becomes negligible in the digital domain.

The first observation is a crucial conclusion for system design because it allows the designer to determine these parameters based on the expected level of cancellation given by the ALMS loop. Furthermore, understanding the relationship among these factors also allows the flexibility in designing the cancellation circuit. For example, if the power of the system is limited, i.e, the gain of the ALMS loop is not high enough, the level of cancellation can still be achieved by a finer tap spacing. In case the size of the ALMS loop is constrained, the loop gain must be increased. The second observation once again states that the performance of the ALMS loop must be considered in the digital domain, and the best level of cancellation given by the ALMS loop is $I S R L B_{d}$.

\section{SIMULATION RESULTS}

To verify the analytical results presented in Section III, simulations are conducted in MATLAB for a single carrier IBFD system9 which uses QPSK modulation and symbol duration $T_{s}=20 \mathrm{~ns}$. The pulse shaping filter and the matched filter are both root raised cosine pulses with the roll-off factor $\beta$. The transmitted power is set to $0 \mathrm{dBm}$ over $50 \mathrm{Ohm}$ load. The transmitted power over $1 \mathrm{Ohm}$ load is found by $0 \mathrm{dBm}+10 \log _{10}(50)=17 \mathrm{dBm}$. Hence, the mean squared amplitude of the transmitted signal for $1 \mathrm{Ohm}$ load is calculated by $V_{X}^{2}=2 \times 10^{(17-30) / 10}=0.1 V^{2}$. The LNA in the receiver is selected with the gain of $\mu=10$. The ALMS loop has the tap spacing $T_{d}=T_{s} / 2$ and the number of taps $L$. The multiplier constants in all the taps are the same and are selected as $K_{1} K_{2}=0.001 V^{2}$. Therefore, the gain of the ALMS loop is $\mu A^{2}=10 \times(0.1 / 0.001)=1000$. The SI power is set to 25 $\mathrm{dB}$ lower than the transmitted signal power.

In the first simulation, the SI channel is chosen as $h(t)=10^{\frac{-25}{20}}\left\{\left[\frac{\sqrt{2}}{2}-0.5 j\right] \delta(t)-0.4 \delta\left(t-0.9 T_{s}\right)+0.3 \delta\left(t-3.3 T_{s}\right)\right\}$, which means that the delays of the reflected paths are fractional of $T_{s}$. The ALMS loop has $L=8$ taps with $T_{s} / 2$ tap spacing. Both pulse shaping filter and matched filter have the roll-off factor of $\beta=0.5$. The power spectrum densities (PSDs) of the baseband equivalent of the SI $Z(t)$, the residual SI in the analog domain $V(t)$, and the residual SI in the digital domain after the matched filter $\tilde{V}(t)$ are presented in Fig. 5. We can see that there are two peaks at the edges of the $V(t)$. However, these peaks are removed in the spectrum of $\tilde{V}(t)$. This simulation confirms the analyses in Section III.B.

In the second simulation, the SI channel has $L$ propagation paths whose coefficients $h_{l}$ are all independent and have a normal distribution with zero-mean. The power delay profile of the channel has an exponential distribution with the root mean square delay spread $\sigma=L T_{s} / 4$. The ISRs at each point of the roll-off factor $\beta$ for different values of $L$ are calculated and 


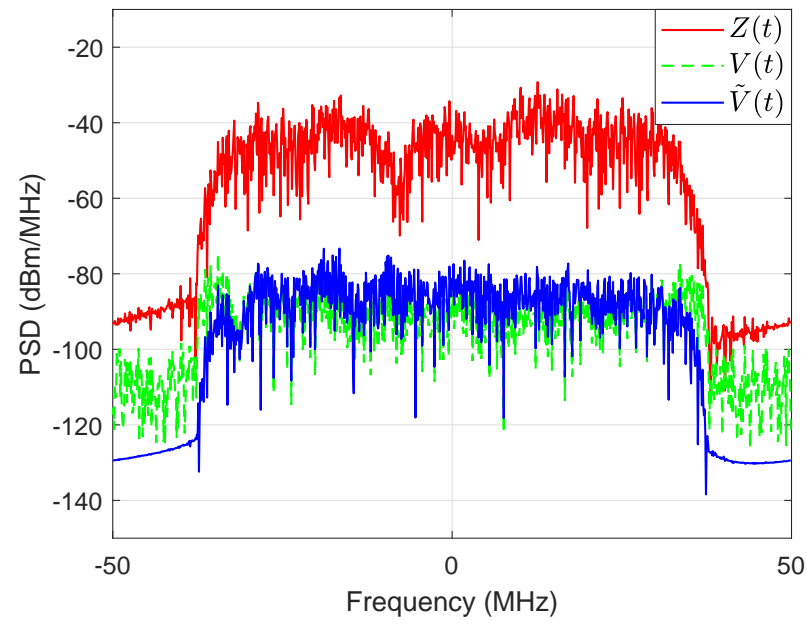

Fig. 5. PSDs of the SI $Z(t)$, residual SI $V(t)$, and residual SI after the matched filter $\tilde{V}(t)$ with $\beta=0.5, \mu A^{2}=1000, T_{d}=T_{S} / 2$, and $L=8$.

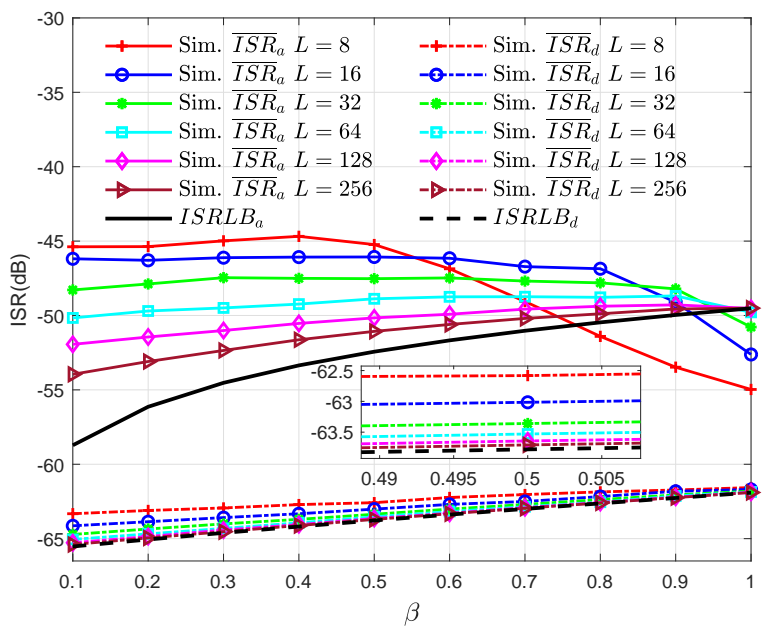

Fig. 6. ISRs in the analog domain and digital domain versus $\beta$ with $\mu A^{2}=$ $1000, T_{d}=T_{s} / 2$.

averaged out over 1000 iterations. The simulated $\overline{I S R}_{a}, \overline{I S R}_{d}$ and their corresponding lower bounds $I S R L B_{a}, I S R L B_{d}$ are presented in Fig. 6 for different values of $L$. The inset shows a closer look of $\overline{I S R}_{d}$. We can see that when $L$ is larger, $\overline{I S R}_{a}$ and $\overline{I S R}_{d}$ are closer to their lower bounds, respectively. This is because the autocorrelation matrix can be well approximated to a circulant matrix and the summation in (29) and (30) approaches the integration when $L$ is sufficiently large. Note that in our analyses, the SI channel is assumed to have the same number of paths as in the ALMS loop. As a result, the SI channels with small number of taps are much shorter compared to those with larger number of taps. Therefore, $\overline{I S R}_{a}$ with smaller $L$ go beyond the lower bound with infinite $L$. However, the matched filter reduces the effects of the SI channel so that $\overline{I S R}_{d}$ are still bounded by $I S R L B_{d}$.

\section{CONClusion}

In this paper, the residual SI powers and the ISRs of an ALMS loop in both analog and digital domains of an IBFD system have been derived using the steady state analysis. The expression of the ISR in the time domain is then converted into the frequency domain by eigenvalue decomposition. From the frequency domain presentation, it is proved that the matched filter has an effect of reducing the peak frequency response of the ALMS loop so that the problem of frequency component enhancement caused by the ALMS loop to the residual SI can be significantly reduced in the digital domain. The corresponding lower bounds of ISRs in both analog and digital domains have also been derived from frequency domain expressions. Comparison between these lower bounds shows that the performance of the ALMS loop should be considered in the digital domain and it is determined by four factors, namely, the loop gain $\mu A^{2}$, the tap delay $T_{d}$, the number of taps $L$, and the roll-off factor $\beta$. The finding of these lower bounds allows the designer to determine the desired level of cancellation given by the ALMS loop. It also provides a room to trade off among these factors to achieve the level of cancellation within given constraints.

\section{ACKNOWLEDGMENT}

This work was supported by the Australian Research Council's Discovery Project Funding Scheme (Project number DP160101693).

\section{APPENDIX A}

PROOF OF CONSTANT $E_{h}\left\{H\left(e^{j \omega_{k}}\right)\right\}$

For SI channels with independent and zero-mean tap coefficients, we prove that $E_{h}\left\{H\left(e^{j \omega_{k}}\right)\right\}$ is a constant for all $k=0,1 \cdots, L-1$ as follow.

$$
\begin{aligned}
E_{h}\left\{\left|H\left(e^{j \omega_{k}}\right)\right|^{2}\right\} & =E_{h}\left\{\sum_{l=0}^{L-1} h_{l} e^{\frac{-j 2 \pi k l}{L}} \sum_{l^{\prime}=0}^{L-1} h_{l^{\prime}}^{*} e^{\frac{j 2 \pi k l^{\prime}}{L}}\right\} \\
& =\sum_{l=0}^{L-1} \sum_{l^{\prime}=0}^{L-1} E_{h}\left\{h_{l} h_{l^{\prime}}^{*}\right\} e^{\frac{-j 2 \pi k\left(l-l^{\prime}\right)}{L}} .
\end{aligned}
$$

Since the SI channel tap coefficients are independent with zero-mean, we have $E_{h}\left\{h_{l} h_{l^{\prime}}^{*}\right\}=0$ for $l \neq l^{\prime}$. Therefore, $E_{h}\left\{\left|H\left(e^{j \omega_{k}}\right)\right|^{2}\right\}=\sum_{l=0}^{L-1} E_{h}\left\{\left|h_{l}\right|^{2}\right\}$ for all $k=0,1 \cdots, L-1$ which is the mean power of the SI channel.

\section{APPENDIX B}

DERIVATION OF ISRLB $B_{a}$ AND ISRLB $B_{d}$

a) ISRLB $B_{a}$ : From $\int_{-\frac{1+\beta}{2 T_{s}}}^{\frac{1+\beta}{2 T_{s}}} P(f) d f=1$ and $T_{d} \leq \frac{T_{s}}{1+\beta}$,

can be simplified as

$$
\begin{aligned}
I S R L B_{a} & =\frac{\int_{-1 / 2 T_{d}}^{1 / 2 T_{d}} \frac{A^{2} P(f)}{\left[1+\mu \frac{A^{2}}{T_{d}} P(f)\right]^{2}} d f}{\int_{-1 / 2 T_{d}}^{1 / 2 T_{d}} A^{2} P(f) d f} \\
& =2 \int_{0}^{\frac{1+\beta}{2 T_{s}}} \frac{P(f)}{\left[1+\mu \frac{A^{2}}{T_{d}} P(f)\right]^{2}} d f .
\end{aligned}
$$


Substituting $P(f)$ from (24) into (36), we have

$$
\begin{gathered}
\int_{0}^{\frac{1+\beta}{2 T_{s}}} \frac{P(f)}{\left[1+\frac{\mu A^{2}}{T_{d}} P(f)\right]^{2}} d f=\int_{0}^{\frac{1-\beta}{2 T_{s}}} \frac{T_{s}}{\left[1+\mu A^{2} \frac{T_{s}}{T_{d}}\right]^{2}} d f \\
+\int_{\frac{1-\beta}{2 T_{s}}}^{\frac{1+\beta}{2 T_{s}}} \frac{\frac{T_{s}}{2}\left[1+\cos \left(\frac{\pi T_{s}}{\beta}\left(f-\frac{1-\beta}{2 T_{s}}\right)\right)\right]}{\left\{1+\mu A^{2} \frac{T_{s}}{2 T_{d}}\left[1+\cos \left(\frac{\pi T_{s}}{\beta}\left(f-\frac{1-\beta}{2 T_{s}}\right)\right)\right]\right\}^{2}} d f .
\end{gathered}
$$

Denoting $a=\mu A^{2} \frac{T_{s}}{T_{d}}$ and $x=\frac{\pi T_{s}}{\beta}\left(f-\frac{1-\beta}{2 T_{s}}\right)$, (37) becomes

$$
\int_{0}^{\frac{1+\beta}{2 T_{s}}} \frac{P(f)}{\left[1+\frac{\mu A^{2}}{T_{d}} P(f)\right]^{2}} d f=\frac{1-\beta}{2(1+a)^{2}}+\frac{\beta}{\pi} \int_{0}^{\pi} \frac{\frac{1}{2}(1+\cos x)}{\left[1+\frac{a}{2}(1+\cos x)\right]^{2}} d x
$$

Defining $t=\tan (x / 2)$ so that $\cos x=\frac{1-t^{2}}{1+t^{2}}$ and $d x=\frac{2 d t}{1+t^{2}}$, we have

$$
\begin{aligned}
\int_{0}^{\pi} \frac{\frac{1}{2}(1+\cos x)}{\left[1+\frac{a}{2}(1+\cos x)\right]^{2}} d x & =2 \int_{0}^{\infty} \frac{1}{\left(t^{2}+a+1\right)^{2}} d t \\
& =\frac{2 \sqrt{a+1}}{(a+1)^{2}} \int_{0}^{\infty} \frac{1}{\left[\left(\frac{t}{\sqrt{a+1}}\right)^{2}+1\right]^{2}} d\left(\frac{t}{\sqrt{a+1}}\right) \\
& =\frac{\pi}{2} \frac{\sqrt{a+1}}{(a+1)^{2}} .
\end{aligned}
$$

Substituting (39) into (38), we obtain the $I S R L B_{a}$ as in (33).

b) $I S R L B_{d}$ : Following the same steps as above, ISRLB $B_{d}$ is derived as

$$
\begin{aligned}
I S R L B_{d} & =\frac{\int_{-1 / 2 T_{d}}^{1 / 2 T_{d}} \frac{A^{2} P^{2}(f)}{\left[1+\mu \frac{A^{2}}{T_{d}} P(f)\right]^{2}} d f}{\int_{-1 / 2 T_{d}}^{1 / 2 T_{d}} A^{2} P^{2}(f) d f} \\
& =\frac{\int_{0}^{\frac{1+\beta}{2 T_{S}}} \frac{P^{2}(f)}{\left[1+\mu \frac{A^{2}}{T_{d}} P(f)\right]^{2}} d f}{\int_{0}^{\frac{1+\beta}{2 T_{S}}} P^{2}(f) d f}
\end{aligned}
$$

Substituting $P(f)$ from (24) into (40) as well as applying the substitution of $x=\frac{\pi T_{s}}{\beta}\left(f-\frac{1-\beta}{2 T_{s}}\right)$ and then $t=\tan (x / 2)$, we have

$$
\begin{aligned}
\int_{0}^{\frac{1+\beta}{2 T_{S}}} \frac{P^{2}(f)}{[1+a P(f)]^{2}} d f & =\frac{T_{s}(1-\beta)}{2(1+a)^{2}}+\frac{T_{s} \beta}{\pi} \int_{0}^{\pi} \frac{\frac{1}{4}(1+\cos x)^{2}}{\left[1+\frac{a}{2}(1+\cos x)\right]^{2}} d x \\
& =\frac{T_{S}(1-\beta)}{2(1+a)^{2}}+\frac{T_{s} \beta}{\pi} \int_{0}^{\infty} \frac{\frac{1}{\left(1+t^{2}\right)^{2}}}{\left(1+a \frac{1}{1+t^{2}}\right)^{2}} \frac{2}{1+t^{2}} d t \\
& =\frac{T_{S}(1-\beta)}{2(1+a)^{2}}+\frac{T_{s} \beta}{\pi} \int_{0}^{\infty} \frac{2}{\left(t^{2}+a+1\right)^{2}\left(t^{2}+1\right)} d t
\end{aligned}
$$

Note that $\frac{2}{\left(t^{2}+a+1\right)^{2}\left(t^{2}+1\right)}$ can be split as

$$
\frac{2}{\left(t^{2}+a+1\right)^{2}\left(t^{2}+1\right)}=\frac{2}{a^{2}}\left[\frac{1}{\left(1+t^{2}\right)}-\frac{1}{\left(t^{2}+a+1\right)}-\frac{a}{\left(t^{2}+a+1\right)^{2}}\right] \text {. }
$$

Therefore, by substituting (42) into (41), we obtain

$$
\begin{gathered}
\int_{0}^{\frac{1+\beta}{2 T_{S}}} \frac{P^{2}(f)}{(1+a P(f))^{2}} d f=\frac{T_{s}(1-\beta)}{2(1+a)^{2}}+\frac{T_{s} \beta}{\pi} \frac{\pi}{a^{2}}\left[1-\frac{1}{\sqrt{a+1}}-\frac{a \sqrt{a+1}}{2(a+1)^{2}}\right] \\
=\frac{T_{s}}{2(1+a)^{2}}\left\{1+\beta\left[\frac{2(a+1)^{2}}{a^{2}}\left(1-\frac{1}{\sqrt{a+1}}-\frac{a \sqrt{a+1}}{2(a+1)^{2}}\right)-1\right]\right\} .
\end{gathered}
$$

The derivation of $\int_{0}^{\frac{1+\beta}{2 T_{S}}} P^{2}(f) d f$ is expressed as

$$
\begin{aligned}
\int_{0}^{\frac{1+\beta}{2 T_{S}}} P^{2}(f) d f & =T_{s} \frac{1-\beta}{2}+\frac{T_{s} \beta}{4 \pi} \int_{0}^{\pi}(1+\cos x)^{2} d x \\
& =\frac{T_{s}}{2}(1-\beta / 4) .
\end{aligned}
$$

From (43) and (44), ISRLB $B_{d}$ is obtained as in (34).

\section{REFERENCES}

[1] A. Sabharwal et al., "In-band full-duplex wireless: Challenges and opportunities," IEEE J. Sel. Areas Commun, vol. 32, no. 9, pp. 16371652, Sept 2014.

[2] Z. Zhang, K. Long, A. V. Vasilakos, and L. Hanzo, "Full-duplex wireless communications: Challenges, solutions, and future research directions," Proceedings of the IEEE, vol. 104, no. 7, pp. 1369-1409, July 2016.

[3] J. Kim et al., "Full-duplex Radios in 5G: Fundamentals, Design and Prototyping," in Signal Processing for 5G. Chichester, UK: John Wiley \& Sons, Ltd, Aug 2016.

[4] J. Choi, M. Jain, and K. Srinivasan, "Achieving single channel, full duplex wireless communication," in Proc. 16th Annu. Int. Conf. Mobi. Com. Chicago, USA, Sept 2010, pp. 1-12.

[5] M. Duarte et al., "Design and characterization of a full-duplex multiantenna system for WIFI networks," IEEE Trans. Veh. Technol., vol. 63, no. 3, pp. 1160-1177, Mar 2014.

[6] T. Oh, Y.-G. Lim, C.-B. Chae, and Y. Lee, "Dual-polarization slot antenna with high cross-polarization discrimination for indoor smallcell mimo systems," IEEE Antennas Wireless Propag. Lett., vol. 14, pp. 374-377, Oct 2014

[7] M. E. Knox, "Single antenna full duplex communications using a common carrier," in Proc. 13th IEEE Annu. Wireless and Microwave Tech. Conf., (WAMICON), Florida, USA, Jun 2012, pp. 1-6.

[8] D. Bharadia, E. McMilin, and S. Katti, "Full duplex radios," Proc. ACM SIGCOMM 2013, vol. 43, no. 4, pp. 375-386, Aug 2013.

[9] D. Korpi et al., "Full-duplex mobile device: Pushing the limits," IEEE Commun. Mag., vol. 54, no. 9, pp. 80-87, Sept 2016.

[10] J. Zhou, T. H. Chuang, T. Dinc, and H. Krishnaswamy, "Integrated wideband self-interference cancellation in the RF domain for FDD and full-duplex wireless," IEEE J. Solid-State Circuits, vol. 50, no. 12, pp. 3015-3031, Dec 2015.

[11] S. B. Venkatakrishnan, E. A. Alwan, and J. L. Volakis, "Wideband RF self-interference cancellation circuit for phased array simultaneous transmit and receive systems," IEEE Access, vol. 6, pp. 3425-3432, Jan 2018.

[12] T. Huusari et al., "Wideband self-adaptive RF cancellation circuit for full-duplex radio: Operating principle and measurements," in Proc. 81st IEEE Veh. Technol. Conf. (VTC Spring), Glasgow, Scotland, 2015, pp. $11-14$.

[13] K. E. Kolodziej, J. G. McMichael, and B. T. Perry, "Multitap RF canceller for in-band full-duplex wireless communications," IEEE Trans. Wireless Commun., vol. 15, no. 6, pp. 4321-4334, June 2016.

[14] D. Liu et al., "On the analog self-interference cancellation for fullduplex communications with imperfect channel state information," IEEE Access, vol. 5, pp. 9277-9290, May 2017.

[15] M. Duarte and A. Sabharwal, "Full-duplex wireless communications using off-the-shelf radios: Feasibility and first results," in Conference Record - Asilomar Conference on Signals, Systems and Computers, California, USA, pp. 1558-1562.

[16] A. Kiayani et al., "Adaptive nonlinear RF cancellation for improved isolation in simultaneous transmit-receive systems," IEEE Trans. Microw. Theory Tech., vol. 66, no. 5, pp. 2299-2312, May 2018.

[17] X. Huang and Y. J. Guo, "Radio frequency self-interference cancellation with analog least mean-square loop," IEEE Trans. Microw. Theory Tech., vol. 65, no. 9, pp. 3336-3350, Sept 2017. 
[18] A. T. Le, L. C. Tran, and X. Huang, "Cyclostationary analysis of analog least mean square loop for self-interference cancellation in in-band fullduplex systems," IEEE Commun. Lett., vol. 21, no. 12, pp. 2738-2741, Sept 2017.

[19] — "On performance of analog least mean square loop for selfinterference cancellation in in-band full-duplex OFDM systems," in Proc. IEEE 85th Veh. Technol. Conf. (VTC Spring), Sydney, Australia, 2017, pp. $1-5$.

[20] T. K. Moon and W. C. Stirling., Mathematical methods and algorithms for signal processing. New Jersey, USA: Prentice Hall, Jun 2000.

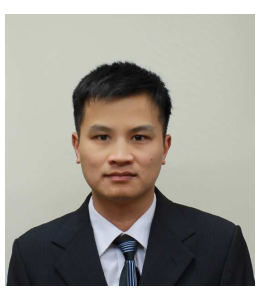

Anh Tuyen Le received the B.Eng and M. Eng degrees in telecommunication engineering from Le Quy Don Technical University, Vietnam and University of Wollongong (UOW), Australia, in 2008 and 2014, respectively. He is currently pursuing the $\mathrm{Ph} . \mathrm{D}$. degree in engineering with the Global Big Data Technologies Center, University of Technology Sydney (UTS), Sydney, NSW, Australia. His research interests include signal processing, circuits and systems for RF and millimetre applications.

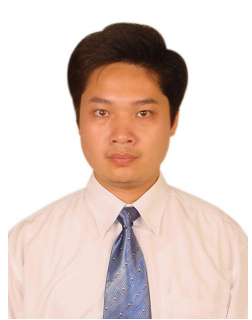

Le Chung Tran (M'09-SM'18) received the B.E. (1st Hon, HD), M.E. (HD), and Ph.D. degrees from University of Transport and Communication (UTC), University of Science and Technology, Vietnam, and University of Wollongong (UOW), Australia, in 1997, 2000, and 2006, respectively, all in Telecommunications Engineering. He was a lecturer at UTC from 1997 to 2012. From 2005 to 2006, he was an associate research fellow at the Wireless Technologies Laboratory, UOW. During 2006-2008, he was a postdoctoral research fellow at University of Luebeck, Germany, under the Alexander von Humboldt $(\mathrm{AvH})$ fellowship. $\mathrm{He}$ has been with the UOW since 2009 to date, where he is currently a senior lecturer. He has achieved the World University Services (WUS) awards (twice), Vietnamese Government's doctoral scholarship, International Postgraduate Research Scholarship (IPRS), and the prestigious Humboldt postdoctoral fellowship. He has served as an advisory board member for Cambridge Scholars Publishing; an editorial board member of the Electrical Engineering: An International Journal (EEIJ); an organizing committee member (Track Chair, Session Chair, Publicity Co-Chair) and a Technical Program Committee (TPC) member for over 30 IEEE conferences. He is a co-author of 75 publications, including one book, which is hold in 337 worldwide libraries. His research interests include 5G, MIMO, space-timefrequency processing, WBANs, IoT, biomedical engineering, ultra-wideband, millimetre wave, cooperative and cognitive communications, software defined radio, network coding, and digital signal processing for communications.

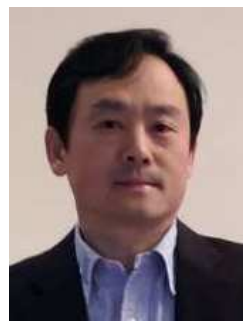

Xiaojing Huang (M'99-SM'11) received the B.Eng., M.Eng., and Ph.D. degrees in electronic engineering from Shanghai Jiao Tong University, Shanghai, China, in 1983, 1986, and 1989, respectively. He was a Principal Research Engineer with the Motorola Australian Research Center, Botany, NSW, Australia, from 1998 to 2003, and an Associate professor with the University of Wollongong, Wollongong, NSW, Australia, from 2004 to 2008. He had been a Principal Research Scientist with the Commonwealth Scientific and Industrial Research Organisation (CSIRO), Sydney, NSW, Australia, and the Project Leader of the CSIRO Microwave and mm-Wave Backhaul projects since 2009. He is currently a Professor of Information and Communications Technology with the School of Electrical and Data Engineering and the Program Leader of Mobile Sensing and Communications with the Global Big Data Technologies Center, University of Technology Sydney (UTS), Sydney, NSW, Australia With over 29 years of combined industrial, academic, and scientific research experience, he has authored over 270 book chapters, refereed journal and conference papers, major commercial research reports, and filed 31 patents.

Prof. Huang was a recipient of the CSIRO Chairman's Medal and the Australian Engineering Innovation Award in 2012 for exceptional research achievements in multigigabit wireless communications.

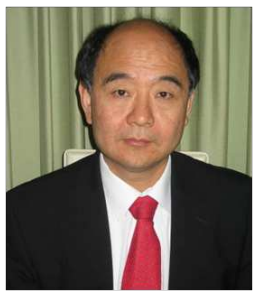

Y. Jay Guo (Fellow'2014) received a Bachelor Degree and a Master Degree from Xidian University in 1982 and 1984, respectively, and a Ph.D Degree from Xian Jiaotong University in 1987, all in China. His research interest includes antennas, mm-wave and $\mathrm{THz}$ communications and sensing systems as well as big data technologies. He has published over 400 research papers and holds 24 patents in antennas and wireless systems. He is a Fellow of the Australian Academy of Engineering and Technology, a Fellow of IEEE and a Fellow of IET, and a member of the College of Experts of Australian Research Council (ARC). He has won a number of most prestigious Australian national awards, and was named one of the most influential engineers in Australia in 2014 and 2015.

Prof Guo is a Distinguished Professor and the founding Director of Global Big Data Technologies Centre at the University of Technology Sydney (UTS), Australia. Prior to this appointment in 2014, he served as a Director in CSIRO for over nine years, directing a number of ICT research portfolios. Before joining CSIRO, he held various senior technology leadership positions in Fujitsu, Siemens and NEC in the U.K.

Prof Guo has chaired numerous international conferences. He was the International Advisory Committee Chair of IEEE VTC2017, General Chair of ISAP2015, iWAT2014 and WPMC'2014, and TPC Chair of 2010 IEEE WCNC, and 2012 and 2007 IEEE ISCIT. He served as Guest Editor of special issues on "Antennas for Satellite Communications" and "Antennas and Propagation Aspects of 60-90GHz Wireless Communications," both in IEEE Transactions on Antennas and Propagation, Special Issue on "Communications Challenges and Dynamics for Unmanned Autonomous Vehicles," IEEE Journal on Selected Areas in Communications (JSAC), and Special Issue on " $5 G$ for Mission Critical Machine Communications", IEEE Network Magazine.

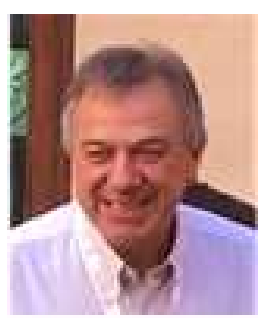

Professor J. (Yiannis) C. Vardaxoglou (F'12) completed his B.Sc. in mathematical physics (1982) and his Ph.D. in Electronics (1985) at the University of Kent, UK. He joined Loughborough University as a lecturer in 1988 and was promoted to Senior Lecturer in 1992 and Professor of Wireless Communications in 1998. He served as the Dean of the School of Electronic, Electrical and Systems Engineering at Loughborough University from 2011-2012. He established the 30 year old Wireless Communications Research (WiCR) group at Loughborough University and founded the Centre for Mobile Communications Research (CMCR). He is the director of Symeta research centre, (www.symeta.co.uk ) funded by an EPSRC Grand Challenge award, researching in a wide-ranging topics applicable to cutting-edge wireless communications technology. His current research focuses primarily on metamaterial structures, additive manufacturing (3D printing) for $\mathrm{RF} / \mathrm{micro} / \mathrm{mm}$ wave engineering. Symeta collaborates with many internationally leading companies and universities. He was recently awarded a prestigious EPSRC's Grand Challenge £5M (FEC) award: Synthesizing 3D Metamaterials for RF, Microwave and THz Applications, (http://gow.epsrc.ac.uk/NGBOViewGrant.aspx?GrantRef=EP/N010493/1 )

He has served as a consultant to various industries, holds six patents and is the Technical Director of Antrum Ltd. He has attracted research funding from industry and has been awarded 18 EPSRC research grants. He has published over 300 refereed journals and conference proceeding papers (with 6500 citations) and has written a book on FSS. He was Chairman of the Executive Committee of the IET's Antennas and Propagation Professional Network in the UK and chaired the IEEE's distinguish lecturer program of the Antennas and Propagation Society (APS) for five years. He founded the Loughborough Antennas \& Propagation Conference (LAPC), which has been running since 2005. He has chaired numerous IEE//IET events and has served on the Steering Committee of the European Conference on Antennas and Propagation, EuCAP. He was the General Chair of EuCAP '07. He was elected a Fellow of the Royal Academy of Engineers in 2011 and a Fellow of the Institute of Electrical and Electronics Engineers in 2012. 